\title{
ABSORCIÓN DE HIERRO EN "MACA" Lepidium meyenii Walp. (Brasicaceae)
}

\section{ABSORPTION OF IRON IN "MACA" Lepidium meyenii Walp. (Brasicaceae)}

\author{
Mery L. Suni ${ }^{1}$, Jorge A. Bravo ${ }^{2}$ y Julio Fabián S. ${ }^{2}$
}

\begin{abstract}
Lepidium meyenii Walp. (Brasicaceae) "maca" is an Andean plant appreciated for its hypocotil which has high medical and nutritional value, and among these, its high iron content. In order to test the possibility of increasing the iron content, an experiment was carried out at greenhouse conditions in Lima, Peru, from May to November 1999, in which solutions containing three levels of natural iron (5, 15 and $25 \mathrm{ppm}$ ) were used to grow maca. Seeds of accession ARB5441 (CIP) were used. The iron content was measured after 50 and 179 days of treatment (dat) by the phenantroline method.
\end{abstract}

Meaningful differences were found in the iron content in leaves and the hypocotil after 50 dat, which increased with the concentration of iron in the solutions, whereas at 179 this trend was noticed only for leaves. The concentration of iron in the hypocotil for each treatment do not increase in time whereas the opposite is observed in the leaves. In general, the concentration of iron obtained in the experiment is higher than that observed in plants grown under natural conditions. The weight of the plant tends to decrease when increasing the concentration of iron in the solution at 50 dat, whereas the opposite trend was observed at $179 \mathrm{ddt}$. The weight of the hypocotils increased noticeably as a function of time, whereas that of the leaves showed only a slight increase.

These results allow us to afirm that a $5 \mathrm{ppm}$ level of iron is adequate and sufficient to obtain high concentrations of iron in the "maca" hypocotils.

Keywords: Lepidium meyenii, "maca", iron, iron absorption, Fe.

\section{Resumen}

Lepidium meyenii Walp (Brasicaceae) "maca" es una planta andina apreciada por sus hipocótilos de alto valor medicinal y nutritivo, entre estos, su elevado contenido de hierro. Para determinar la posibilidad de incrementarlo se llevó a cabo un experimento en invernadero, en Lima, durante el periodo de mayo a noviembre de 1999, en el cual se proporcionó tres niveles de hierro natural $(5,15$ y 25 ppm) en soluciones nutritivas. Se utilizaron semillas, entrada ARB5441 (CIP). Se determinó el contenido de hierro a los 50 y 179 días después del tratamiento (ddt) por el método de fenantrolina.

Se encontró diferencias significativas en el contenido de hierro en hojas e hipocótilo a los 50 ddt, incrementado este en relación a la cantidad de hierro suministrada, mientras que a los 179 ddt esta misma respuesta se observa sólo a nivel de hojas. La concentración de hierro obtenida en el hipocótilo para cada tratamiento no incrementó en el tiempo mientras que en la hoja sí. En general los valores de concentración de hierro obtenidos son mayores a los reportados en la literatura. El peso de la planta tendió a disminuir en relación al nivel de hierro a los $50 \mathrm{ddt}$, pero a los 179 ddt tendió a incrementar. En función al tiempo, el peso de los hipocótilos en cada tratamiento incrementó notablemente; mientras que las hojas, ligeramente.

Los resultados obtenidos nos permiten señalar que un nivel de $5 \mathrm{ppm}$ de hierro resulta adecuado y suficiente para obtener concentraciones altas de hierro en los hipocótilos de "maca".

Palabras clave: Lepidium meyenii, "maca", hierro, absorción de hierro, Fe.

\footnotetext{
1 Laboratorio de Fisiología Vegetal, Facultad de Ciencias Biológicas, UNMSM, E-mail: msunin@unmsm.edu.pe, Fax: 14649110.

2 Laboratorio de Suelos. Facultad de Ciencias Físicas, UNMSM. E-mail: jbravoc@unmsm.edu.pe, Fax: 14521343.
} 


\section{Introducción}

Lepidium meyenii Walp., maca, es una planta altoandina apreciada por sus hipocótilos de valor nutritivo y medicinal. El alto valor nutritivo de la maca la convierte en un cultivo alimenticio de gran potencial, no sólo por su contenido de proteínas y carbohidratos, sino también por el contenido de minerales como Fe, Ca, Cu y Zn (Dini et al., 1994; Tello et al. 1992; Arbizu y Holle 1994). Es precisamente su alto contenido de hierro en comparación con otras raíces y tubérculos andinos lo que motiva el interés del presente estudio. El hierro, un elemento importante tanto para la planta misma como para los que la consumen, es absorbido del suelo por las raíces y transportado a otros órganos de la planta donde se usa de inmediato o puede acumularse (Brown, 1978). El hierro forma parte de muchas enzimas que participan en la fotosíntesis y otras rutas metabólicas (Taiz and Zeiger, 1991). Además, el hierro es indispensable en la nutrición humana para prevenir la anemia y la desnutrición (American Academy of Family Physicians, 2000). Por otro lado, pese a que el contenido de hierro de los suelos donde se desarrolla la maca puede tener valores que fluctúan de 34 a 124 ppm (Alaluna, comunicación personal), la mayor parte del hierro en el suelo se encuentra predominantemente en una forma química no asimilable por la planta (Shulte, 1992), de modo tal que brindándole el hierro en la forma apropiada se podría lograr incrementar el contenido de este elemento en la planta y elevar su calidad nutricional.

Por este motivo el presente trabajo tiene por objetivo determinar el efecto de dosis crecientes de hierro $(5,15$ y 25 ppm) en el contenido de este elemento en las hojas e hipocótilo de la maca.

\section{Materiales y métodos}

El cultivo de las plantas de maca utilizadas en el presente experimento se realizó bajo condiciones de invernadero en el Laboratorio de Fisiología Vegetal de la Facultad de Cien- cias Biológicas de la Universidad Nacional Mayor de San Marcos, Lima, Perú, entre los meses de mayo y noviembre de 1999. Se suministró los diferentes niveles de hierro mediante soluciones nutritivas y evaluadas mediante el método de fenantrolina.

\section{Material Biológico}

Las plantas evaluadas, distribuidas en un diseño completo al azar, fueron obtenidas a partir de semillas de maca, Lepidium meyenii entrada ARB5441 mantenida en el banco de germoplasma de este cultivo por el Centro Internacional de la Papa, Lima, Perú.

\section{Materiales en general}

Para el cultivo de las plantas: macetas, arena de cuarzo, solución nutritiva San Marcos y hierro natural quelado (EDDHA) para la variación de la cantidad de hierro en la solución.

Para la cuantificación de hierro: ácido sulfúrico, peróxido de hidrógeno, reactivo fenantrolina (Ferrover).

Para análisis químico: espectrofotómetro $\mathrm{HACH} \mathrm{DR} / 2010$, digestor HACH.

\section{Métodos}

\section{Procedimientos generales}

Periodo de cultivo: se sembró el 3 de mayo de 1999, se inició el riego con los tratamientos de hierro el 28 de mayo y se finalizó el 22 de noviembre del mismo año, con un total de 179 días de tratamiento (ddt).

El riego consistió en $300 \mathrm{ml}$ de la solución de tratamiento por planta por semana.

Se utilizó la solución nutritiva con tres niveles de concentración de hierro: 5, 15 y 25 ppm (estimados nominales según cálculos teóricos). Estas concentraciones de hierro fueron determinadas también por el método de fenatrolina y correspondieron a 5, 17 y 29 ppm respectivamente.

La primera evaluación se realizó a los 50 ddt y la segunda a los 179 ddt. Consistió en determinar el peso de las muestras en estado 


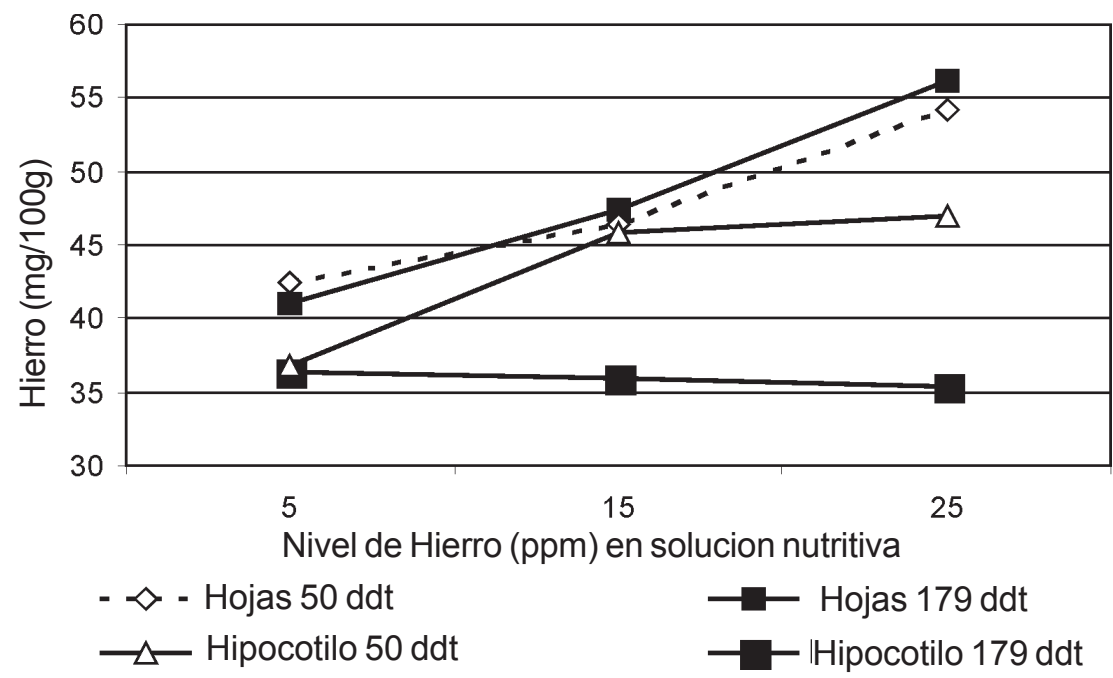

Figura 3. Absorción y distribución de hierro en Lepidium meyenii frente a tres niveles de este elemento, expresado en mg/100 g en hojas e hipocótilo.

seco, y el contenido de Fe en las hojas e hipocótilo de las plantas.

\section{Evaluación del peso y contenido de hie- rro}

Para el análisis de hierro las hojas e hipocótilo fueron secadas en estufa de aire forzado a $40{ }^{\circ} \mathrm{C}$, luego llevadas a un desecador y pulverizadas en un mortero. Para la digestión de cada muestra se siguió el protocolo para hierro en muestras sólidas del Manual del Digestor Hach. Para la cuantificación de hierro se siguió el método FerroVer (Método 8008, adaptado del Standard Methods for the Examination of Water and Wastewater) obteniéndose el contenido de hierro en $\mathrm{mg} / 100$ $\mathrm{g}$ de materia seca.

\section{Resultados}

\section{Contenido de hierro}

Los resultados del contenido de hierro por órgano, tratamiento y fecha de evaluación se presentan en la Tabla 1 y Figura 1. En las ho-

Tabla 1. Contenido de Fe en $\mathrm{mg} / 100 \mathrm{~g}$ de materia seca, según órgano y tratamiento medidos por el método de fenantrolina a los 50 y 179 días después del tratamiento (ddt). Los valores a los $50 \mathrm{ddt}$ corresponden al promedio de 2 repeticiones y para a $179 \mathrm{ddt}$ a 4 repeticiones.

\begin{tabular}{c|cc|cc}
\hline & \multicolumn{2}{|c|}{ Hojas } & \multicolumn{2}{c}{ Hipocótilo } \\
\hline ppm & $50 \mathrm{ddt}$ & $179 \mathrm{ddt}$ & $50 \mathrm{ddt}$ & $179 \mathrm{ddt}$ \\
\hline 5 & $42,4 \mathrm{c}$ & $41,1 \mathrm{c}$ & $36,8 \mathrm{~b}$ & $36,3 \mathrm{a}$ \\
15 & $46,4 \mathrm{~b}$ & $47,4 \mathrm{~b}$ & $45,8 \mathrm{a}$ & $35,9 \mathrm{a}$ \\
25 & $54,2 \mathrm{a}$ & $56,2 \mathrm{a}$ & $47,0 \mathrm{a}$ & $35,4 \mathrm{a}$ \\
\hline $\operatorname{LSD}(0,05)$ & 1,83 & 1,71 & 1,75 & $1,27(\mathrm{~ns})$ \\
\hline
\end{tabular}


Tabla 2. Peso seco (en g/planta) según órgano y tratamiento a los 50 y 179 ddt. Los valores a los 50 ddt corresponden al promedio de 3 repeticiones y para a 179 ddt a 2 repeticiones.

\begin{tabular}{c|cc|cc}
\hline & \multicolumn{2}{|c|}{ Hojas } & \multicolumn{2}{c}{ Hipocótilo } \\
\hline ppm & $50 \mathrm{ddt}$ & $179 \mathrm{ddt}$ & $50 \mathrm{ddt}$ & $179 \mathrm{ddt}$ \\
\hline 5 & $1,377 \mathrm{a}$ & $1,160 \mathrm{a}$ & $0,270 \mathrm{a}$ & $4,455 \mathrm{a}$ \\
15 & $1,157 \mathrm{a}$ & $1,680 \mathrm{a}$ & $0,117 \mathrm{a}$ & $5,450 \mathrm{a}$ \\
25 & $0,843 \mathrm{a}$ & $1,765 \mathrm{a}$ & $0,076 \mathrm{a}$ & $4,440 \mathrm{a}$ \\
\hline LSD $(0,05)$ & $0,68(\mathrm{~ns})$ & $1,28(\mathrm{~ns})$ & $0,22(\mathrm{~ns})$ & $3,66(\mathrm{~ns})$ \\
\hline
\end{tabular}

jas se observa diferencias significativas en el contenido de hierro a los 50 y 179 ddt, incrementándose en relación directa a la mayor cantidad de Fe suministrada y mayor tiempo. Mientras que en los hipocótilos se observó diferencias significativas sólo a los $50 \mathrm{ddt}$, incrementándose en relación a la cantidad de Fe suministrada y a los 179 ddt una ligera disminución en comparación con la primera evaluación.

\section{Peso seco}

Al evaluar, a los 179 ddt, el efecto de los tratamientos sobre el peso de la planta, Tabla 2 , se encontró una tendencia a que éste se incremente con el nivel de Fe suministrado en la solución. El peso de los hipocótilos se incrementó en mayor proporción en función al tiempo, mientras que el de las hojas se incrementó ligeramente. A los 50 ddt, la tendencia a mayor dosis de Fe suministrado era más bien de disminución del peso seco de las hojas e hipocótilo, tal vez como respuesta inicial a las dosis elevadas de Fe.

\section{Discusión y conclusión}

En el presente trabajo se han obtenido valores de contenido de hierro (Tabla 1) mayores que los reportados en la literatura lo cual puede deberse al suministro continuo de hierro en forma soluble brindado. Garay (1996) señala que en las hojas de maca hay 0,248 $\mathrm{mg} / \mathrm{g}$ pero no indica el ecotipo ni el método de análisis utilizado. Y los valores que se reportan para el hipocótilo son $0,166 \mathrm{mg} / \mathrm{g}$ en materia secada al aire (Dini et al., 1994), 14,7 mg/ 100 g (Castro, 1995) y 0,154 mg/g (Solís y Trigos (1995).

Asimismo todos estos valores de contenido de hierro en el hipocótilo de maca son superiores a los encontrados en otras raíces y tubérculos andinos (Dini et al., 1994 y Tapia, 1997) y no pueden incrementarse más aunque se le suministre dosis altas de hierro asimilable durante el cultivo de la planta como se muestran en la Tabla 1 para hipocótilo. En cambio para sus hojas la concentración de Fe incrementa a los $179 \mathrm{ddt}$, a diferencia del hipocótilo, donde disminuye. Esto está indicando la buena absorción del elemento y 
removilización hacia las hojas como se observa en las plantas hiperacumuladoras, tolerantes al alto contenido de iones en su medio (Lasat y Kochian, 1997).

Finalmente, se puede indicar que un suministro de 5 ppm de hierro absorbible permite obtener hipocótilos y en general una planta con alto contenido de hierro, por lo que este nivel de tratamiento de hierro parece resultar suficiente para obtener concentraciones altas de hierro en los hipocótilos de maca.

\section{Reconocimientos}

Este trabajo se realizó con el apoyo del Proyecto "Andean Root and Tuber Crops: Realizing the Promise of Forgotten Foods" de la Fundación McKnight.

\section{Literatura citada}

American Academy of Family Physicians. 2000. Anemia: Cuando la causa es la falta de hierro. http://familydoctor.org/spanish/e009.html. Acceso: Setiembre 2002

Brown, J. 1978. Mechanism of iron uptake by plants. Plant, Cell and Environment (1): 249-257.

Castro, G. 1995. Producción de Lepidium meyenii Walp (MACA), en dos sistemas de siembra. Meseta de Bombón. I Congreso Peruano de Cultivos Andinos. Ayacucho (Perú). 11-16 Septiembre 1995. Universidad Nacional de San Cristóbal de Huamanga. Programa de Investigación en Cultivos Andinos (Perú). 5(1): 33-34.
Dini, A.; G. Migliuolo; L. Rastrelli; P. Saturnino; O. Schettino, 1994. Chemical composition of Lepidium meyenii. Food Chemistry 49, 347349.

Garay, O. 1996. Resúmenes del Primer curso sobre el cultivo de maca. INIA.

Lasat, M. M. y L. V. Kochian. 1997. Physiological basis for $\mathrm{Zn}$ hyperaccumulation in Tlaspi caerulscens. En: Radical Biology:Advances and Perspectives on the Function of Plant Roots, pp. 139-149, HE Flores, JP Lynch, D Eissenstat, eds. American Society of Plant Physiologists.

Shulte, E. E. 1992. Soil and Applied Iron. Cooperative Extension Publications, University of Wisconsin-Extension.

http://www 1.uwex.edu/ces/pubs/pdf/ A3554.PDF. Acceso: Marzo 2002.

Solís, R.; M. Trigos. 1995. Valor nutricional, morfología y clasificación de las especies de maca en la zona altoandina de Pasco. 1. Congreso Peruano de Cultivos Andinos. Ayacucho (Perú) 1116 septiembre 1995. Cultivos Andinos, Universidad Nacional de San Cristóbal de Huamanga. Programa de Investigación en Cultivos Andinos (Perú). 5(1): 69-70.

Tapia, M. E. 1997. Cultivos andinos subexplotados y su aporte a la alimentación. Segunda edición. FAO. Oficina Regional de la FAO para América Latina y el Caribe. Chile.

Tello, J., M. Hermann y A. Calderón. 1992. La maca (Lepidium meyenii Walp): Cultivo alimenticio potencial para las zonas altoandinas. Boletín de Lima, N. ${ }^{\circ} 81$, pp. 59-66. 